\title{
Supplementary material to 'The impact of differences in large-scale circulation output from climate models on the regional modeling of ozone and PM'
}

\section{Statistical evaluation}

Table S1a. Annual average behavior of temperatures for the period 1989-2009. Standard deviations represent the day-to-day variability. Numbers in italics indicate that the difference with RLE_ERA is not significant at the $\mathrm{P}=0.05$ level.

\begin{tabular}{|c|c|c|c|c|c|c|c|c|c|c|c|c|}
\hline Station & RLE_ER & & & & RLE_ECH & & & & RLE_MI & $\mathrm{OC}$ & & \\
\hline & $\# \mathrm{~T}>25$ & $\# \mathrm{~T}<5$ & $\mathrm{~T}_{\max }$ & std & $\# \mathrm{~T}>25$ & $\# \mathrm{~T}<5$ & $\mathrm{~T}_{\max }$ & Std & $\# \mathrm{~T}>25$ & $\# \mathrm{~T}<5$ & $\mathrm{~T}_{\max }$ & std \\
\hline Vredepeel & 28 & 54 & 13.6 & 7.9 & 16 & 46 & 13.1 & 7.0 & 30 & 44 & 14.1 & 7.7 \\
\hline Sniezka & 30 & 105 & 11.9 & 9.4 & 17 & 96 & 11.6 & 8.5 & 29 & 101 & 12.2 & 9.1 \\
\hline Melpitz & 37 & 81 & 13.1 & 9.1 & 20 & 73 & 12.6 & 8.2 & 39 & 74 & 13.6 & 8.9 \\
\hline Paris & 37 & 41 & 14.6 & 7.9 & 25 & 35 & 14.1 & 7.1 & 29 & 33 & 14.6 & 7.3 \\
\hline Els Torms & 126 & 1 & 21.4 & 8.5 & 124 & 1 & 21.2 & 8.2 & 99 & 1 & 20.1 & 7.00 \\
\hline Madrid & 109 & 6 & 19.2 & 9.3 & 105 & 7 & 18.6 & 9.4 & 76 & 4 & 17.4 & 7.9 \\
\hline Montelibretti & 63 & 0 & 18.3 & 5.9 & 19 & 0 & 17.2 & 5.0 & 55 & 0 & 18.2 & 5.5 \\
\hline
\end{tabular}

Table S1b. Annual average behavior of rain 1989-2009. Wet days are days with more than $0.5 \mathrm{~mm}$ rain.

\begin{tabular}{|l|rr|rr|rr|}
\hline Station & \multicolumn{2}{|l|}{ RLE_ERA } & \multicolumn{2}{|l|}{ RLE_ECHAM } & \multicolumn{2}{l|}{ RLE_MIROC } \\
\hline & wet days & yearly rain $(\mathrm{mm})$ & wet days & yearly rain $(\mathrm{mm})$ & wet days & yearly rain (mm) \\
\hline Vredepeel & 196 & 748 & 227 & 956 & 199 & 752 \\
Sniezka & 184 & 653 & 212 & 803 & 198 & 708 \\
Melpitz & 168 & 533 & 194 & 668 & 175 & 584 \\
Paris & 179 & 690 & 212 & 872 & 201 & 759 \\
Els Torms & 71 & 336 & 80 & 383 & 93 & 453 \\
Madrid & 93 & 431 & 124 & 675 & 116 & 544 \\
Montelibretti & 98 & 482 & 109 & 595 & 137 & 803 \\
\hline
\end{tabular}


Table S1c. Annual averages wind speed 1989-2009

\begin{tabular}{|l|r|r|rr|r|r|}
\hline Station & \multicolumn{2}{|l|}{ RLE_ERA } & \multicolumn{2}{l|}{ RLE_ECHAM } & \multicolumn{2}{l|}{ RLE_MIROC } \\
\hline & $\#_{\mathrm{v}<2 \mathrm{~m} / \mathrm{s}}$ & average $\mathrm{v}$ & $\# \mathrm{v}<2 \mathrm{~m} / \mathrm{s}$ & average $\mathrm{v}$ & $\# \mathrm{v}<2 \mathrm{~m} / \mathrm{s}$ & average $\mathrm{v}$ \\
\hline Vredepeel & 40 & 4.0 & 32 & 4.3 & 44 & 3.9 \\
Sniezka & 55 & 3.2 & 48 & 3.4 & 66 & 3.1 \\
Melpitz & 34 & 4.0 & 28 & 4.2 & 41 & 3.8 \\
Paris & 26 & 3.9 & 23 & 4.2 & 31 & 3.8 \\
Els Torms & 123 & 2.8 & 101 & 3.1 & 125 & 2.9 \\
Madrid & 64 & 3.0 & 52 & 3.4 & 67 & 3.1 \\
Montelibretti & 34 & 3.9 & 30 & 4.4 & 40 & 4.0 \\
\hline
\end{tabular}

Table S2a. Average behavior of temperature, 2041-2060. Differences with respect to the present-day simulation are significant for all stations

\begin{tabular}{|l|r|r|r|r|r|r|r|r|}
\hline Station & \multicolumn{1}{|l|}{ RLE_ECHAM } & \multicolumn{1}{|l|}{ RLE_MIROC } \\
\hline & \# T $>25 \mathrm{C}$ & \# T $<5 \mathrm{C}$ & $\mathrm{T}_{\max }$ & std & \# T $>25 \mathrm{C}$ & \# T $<5 \mathrm{C}$ & $\mathrm{T}_{\max }$ & Std \\
\hline Vredepeel & 25 & 33 & 14.3 & 7.1 & 55 & 21 & 16.3 & 7.7 \\
Sniezka & 26 & 76 & 12.8 & 8.4 & 53 & 65 & 14.4 & 9.0 \\
Melpitz & 27 & 53 & 13.8 & 8.0 & 65 & 42 & 15.7 & 8.7 \\
Paris & 38 & 21 & 15.2 & 7.3 & 65 & 12 & 17.0 & 7.5 \\
Els Torms & 148 & 0 & 22.9 & 8.6 & 145 & 0 & 23.2 & 7.5 \\
Madrid & 129 & 2 & 20.7 & 10.0 & 123 & 0 & 21.0 & 8.8 \\
Montelibretti & 59 & 0 & 18.5 & 5.3 & 102 & 0 & 20.8 & 5.8 \\
\hline
\end{tabular}

Table S2b. Average rain 2041-2060. Wet days are days with more than $0.5 \mathrm{~mm}$ rain

\begin{tabular}{|l|rr|rr|}
\hline Station & \multicolumn{3}{|l|}{ RLE_ECHAM } & \multicolumn{3}{|c|}{ RLE_MIROC } \\
\hline & wet days & yearly rain $(\mathrm{mm})$ & wet days & yearly rain $(\mathrm{mm})$ \\
\hline Vredepeel & 228 & 993 & 199 & 832 \\
Sniezka & 219 & 869 & 202 & 770 \\
Melpitz & 200 & 745 & 177 & 635 \\
Paris & 210 & 888 & 193 & 788 \\
Els Torms & 74 & 336 & 87 & 394 \\
Madrid & 104 & 617 & 97 & 464 \\
Montelibretti & 103 & 610 & 129 & 759 \\
\hline
\end{tabular}


Table S2c. Average wind speed 2041-2060.

\begin{tabular}{|l|r|r|r|r|}
\hline Station & \multicolumn{2}{|l|}{ RLE_ECHAM } & \multicolumn{2}{|l|}{ RLE_MIROC } \\
\hline & $\# \mathrm{v}<2 \mathrm{~m} / \mathrm{s}$ & average $\mathrm{v}$ & $\# \mathrm{v}<2 \mathrm{~m} / \mathrm{s}$ & average $\mathrm{v}$ \\
\hline Vredepeel & 31 & 4.3 & 50 & 3.8 \\
Sniezka & 47 & 3.4 & 68 & 3.0 \\
Melpitz & 31 & 4.2 & 47 & 3.7 \\
Paris & 23 & 4.2 & 34 & 3.7 \\
Els Torms & 99 & 3.2 & 123 & 2.9 \\
Madrid & 54 & 3.4 & 61 & 3.1 \\
Montelibretti & 26 & 4.4 & 41 & 4.0 \\
\hline
\end{tabular}

Table S3. Summer (JJA) average daily maximum $\mathrm{O}_{3}$ concentrations and annual average PM10 concentrations, present-day and future period for each of the model runs. Standard deviations represent the day to day variability.

\begin{tabular}{|l|r|r|r|r|}
\hline \multicolumn{1}{|l|}{ RLE_ERA (1989-2009) } & & PM10 & \\
\hline & $\mathrm{O}_{3 \max }$ & stdev & average & stdev \\
\hline Station & average & 28 & 13.6 & 6.3 \\
\hline Vredepeel & 100 & 18 & 8.7 & 4.3 \\
Sniezka & 108 & 20 & 8.7 & 4.3 \\
Melpitz & 107 & 26 & 36.3 & 20.9 \\
Paris & 99 & 19 & 8.9 & 4.8 \\
Els Torms & 118 & 15 & 17.8 & 7.4 \\
Madrid & 98 & 17 & 10.8 & 4.3 \\
Montelibretti & 123 & & & \\
\hline
\end{tabular}

\begin{tabular}{|l|r|r|r|r|r|r|r|r|r|r|}
\hline RLE_ECHAM & \multicolumn{9}{|l|}{$(1989-2009)$} & \multicolumn{2}{|l|}{$(2041-2060)$} & \multicolumn{2}{l|}{ Relative change \% } \\
\hline & \multicolumn{2}{|c|}{$\mathrm{O}_{3 \max }$} & \multicolumn{2}{|c|}{ PM10 } & \multicolumn{2}{|c|}{$\mathrm{O}_{3 \max }$} & \multicolumn{2}{|c|}{ PM10 } & & \\
\hline station & average & stdev & average & stdev & average & stdev & average & stdev & $\mathrm{O}_{3}$ & PM10 \\
\hline Vredepeel & 91 & 24 & 12.6 & 5.9 & 93 & 27 & 12.6 & 6.0 & +2.9 & +0.5 \\
Sniezka & 102 & 17 & 8.2 & 4.1 & 106 & 20 & 7.8 & 3.9 & +3.2 & -4.2 \\
Melpitz & 100 & 19 & 8.2 & 4.1 & 103 & 21 & 8.0 & 4.0 & +2.7 & -2.7 \\
Paris & 91 & 24 & 32.3 & 20.1 & 96 & 27 & 31.7 & 19.6 & +6.1 & -2.0 \\
Els Torms & 113 & 19 & 7.6 & 4.4 & 119 & 21 & 7.8 & 4.5 & +5.0 & +2.6 \\
Madrid & 98 & 15 & 15.3 & 7.4 & 102 & 16 & 15.7 & 7.5 & +4.0 & +2.3 \\
Montelibretti & 120 & 17 & 10.1 & 4.0 & 124 & 19 & 10.0 & 4.1 & +3.4 & -0.4 \\
\hline
\end{tabular}




\begin{tabular}{|l|r|r|r|r|r|r|r|r|r|r|}
\hline RLE_MIROC & \multicolumn{9}{|l|}{$(1989-2009)$} & \multicolumn{2}{l|}{$(2041-2060)$} & \multicolumn{2}{l|}{ Relative change $\%$} \\
\hline & \multicolumn{2}{|c|}{$\mathrm{O}_{3 \max }$} & \multicolumn{2}{|c|}{ PM10 } & \multicolumn{2}{|c|}{$\mathrm{O}_{3 \max }$} & \multicolumn{2}{|c|}{ PM10 } & & \\
\hline station & average & stdev & average & stdev & average & stdev & average & stdev & $\mathrm{O}_{3}$ & PM10 \\
\hline Vredepeel & 107 & 26 & 13.8 & 6.4 & 115 & 29 & 14.5 & 7.2 & +7.7 & +4.6 \\
Sniezka & 110 & 16 & 8.6 & 4.2 & 116 & 18 & 8.4 & 4.3 & +5.6 & -2.0 \\
Melpitz & 111 & 19 & 8.5 & 4.3 & 117 & 20 & 8.5 & 4.6 & +5.2 & +0.2 \\
Paris & 105 & 27 & 35.6 & 20.4 & 113 & 30 & 36.4 & 19.7 & +6.9 & +2.0 \\
Els Torms & 115 & 18 & 8.0 & 4.5 & 122 & 19 & 8.2 & 4.6 & +6.7 & +0.1 \\
Madrid & 96 & 15 & 17.3 & 7.5 & 101 & 16 & 17.3 & 7.4 & +4.8 & +0.2 \\
Montelibretti & 123 & 17 & 10.1 & 4.2 & 130 & 12 & 10.3 & 4.3 & +5.8 & +1.6 \\
\hline
\end{tabular}



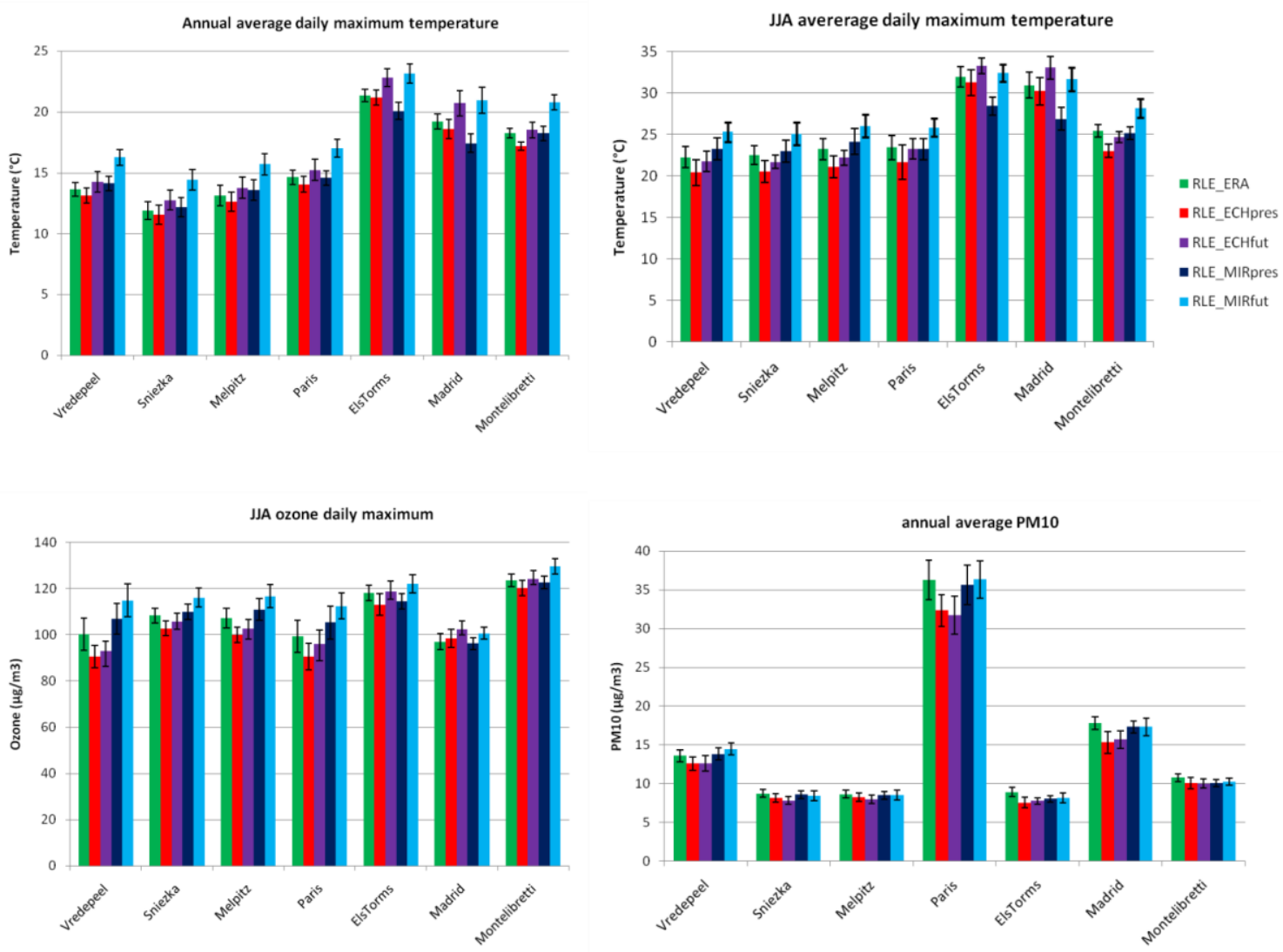

Figure S1.Average values for daily maximum temperature (annual and summer averages) and PM10 (annual) and ozone concentrations (summer averages), with standard deviations to indicate the interannual variability. 

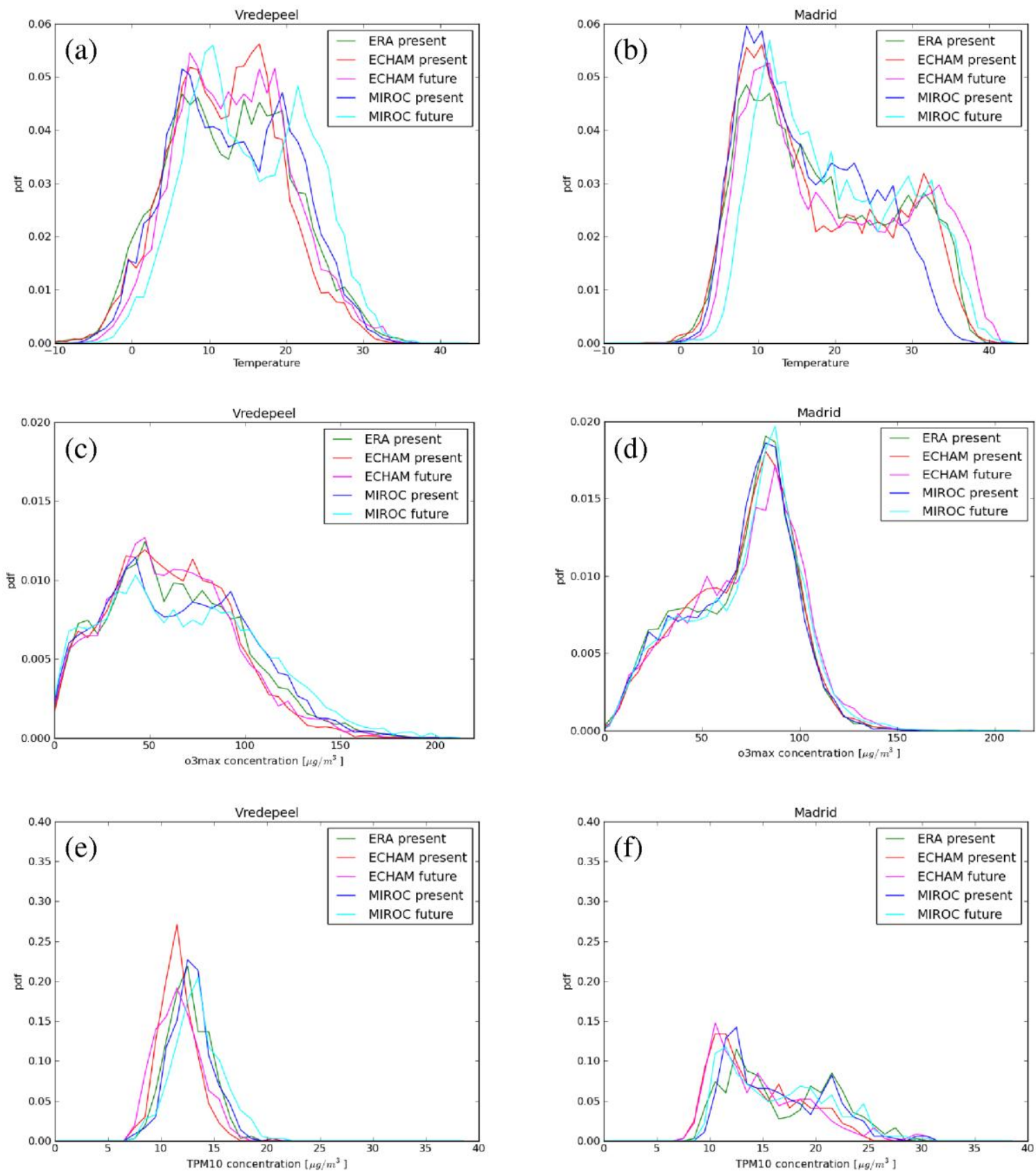

Figure S2. Histogram plots (probability distribution) of daily maximum temperature $(a, b)$, daily maximum ozone concentration, not restricted to summer (c,d) and daily average total PM10 concentration(e,f) for Vredepeel (left) and Madrid (right). 